\title{
Dynamics of 2SIH2R Rumor-Spreading Model in a Heterogeneous Network
}

\author{
Yan Wang $\mathbb{D}^{1,2}$ Feng Qing $\mathbb{D}^{1,2}$ and Ming Yan $\mathbb{D}^{3}$ \\ ${ }^{1}$ School of Data Science and Intelligent Media, Communication University of China, Beijing 100024, China \\ ${ }^{2}$ State Key Laboratory of Media Convergence and Communication, Communication University of China, Beijing 100024, China \\ ${ }^{3}$ School of Information and Communications Engineering, Communication University of China, Beijing 100024, China
}

Correspondence should be addressed to Ming Yan; yanm@cuc.edu.cn

Received 10 July 2021; Revised 7 October 2021; Accepted 15 December 2021; Published 17 January 2022

Academic Editor: Enrico M. Vitucci

Copyright (c) 2022 Yan Wang et al. This is an open access article distributed under the Creative Commons Attribution License, which permits unrestricted use, distribution, and reproduction in any medium, provided the original work is properly cited.

Due to the development of social media, the threshold for information dissemination has become lower than ever before. As a special kind of information, rumors are usually harmful and are usually accompanied by a high degree of ambiguity that makes them difficult to immediately identify, but "rumors stop at wise men." When someone identifies a rumor as false and begins spreading the truth instead, a confrontational relationship obtains between the rumor and the truth that leads to the stifling of the former. Given this, we developed a 2SIH2R model in this study that contains mechanisms of discernment and confrontation in a heterogeneous network to examine the dissemination of the rumor and the truth. By using mean-field equations of the 2SIH2R model, the threshold of the spreading of each can be determined separately in three cases. The results of a numerical simulation show that under the same conditions, the greater is the mechanism of discernment or confrontation, the smaller is the instantaneous maximum influence and the final range of influence of the rumor. It can be also concluded that the earlier release of the truth about the event by the government can significantly control the rumor. Secondly, it is more effective to publish the truth in advance than after the rumor has appeared. Thirdly, it is more important for the government to increase education and improve the ability of citizens to reveal the rumor than to increase the spread of the truth after the rumor occurs. These results can be used to help reduce the harmful effects of rumors.

\section{Introduction}

With the advancement of the Internet and social network services (SNS) such as WeChat and Facebook, the speed, efficiency, and range of information dissemination have significantly improved compared with the era of word of mouth. As a special kind of information, rumors usually negatively affect people's psychology and behavior, may damage the government's credibility, cause social shocks, endanger public safety, and harm public interests [1-3]. The impact of rumors is especially significant nowadays because they can be spread more quickly and widely through SNS. Therefore, it is necessary to study the phenomenon of spreading rumors.

Rumors are special information that has not been officially confirmed, and that might be eventually found as true or false $[4,5]$. But in this research, we assume that the rumors are false. In 1947, Allport and Postman [6] have proposed a quantitative formula to describe the spread of rumors:rumors $=i \times a$ (where $i$ and $a$ represent the importance of the given information and the degree of unknowability of the relevant event). This formula suggests that when a rumor has a certain importance-that is, $i$ is not equal to zero-the higher the degree of indiscernibility of the rumor is, the greater is its impact. Rumors that can be disseminated have a higher degree of indiscernibility so that they cannot be immediately identified as such. Although rumors are confusing and have a large impact over a short period of time, an ancient Chinese idiom holds that "rumors stop at wise men." When someone identifies a rumor and may begin spreading the truth, a confrontational relationship obtains between the rumor and the truth [7] that leads to the former being stifled. Therefore, establishing a model based on this idea will better simulate the spreading process 
of rumors and study the influence of various factors on this process. Through this model, we can study how to reduce the spread of rumors and expand the spread of truth; how does the time interval between the appearance of the truth and the rumors affect the entire spreading process, etc. so that the government can take corresponding measures to reduce the possible impact of rumors.

In light of the apparent similarities between the transmission of epidemic diseases and the spread of rumors, models for the spread of the former are widely used to represent the dissemination of the latter [8]. Daley and Kendall $[9,10]$ proposed the first rumor spreading model, called the DK model, that divides a given population into three categories (the people who have not the heard rumor; the people who actively spreading rumor; the people who no longer spreading rumor). The three categories correspond to "susceptible," "infective," and "recovered" in the SIR epidemic model, respectively. In the DK model, when two individuals in different groups come into contact, they transfer their identities with a certain probability. The most significant shortcoming of the DK model is that it ignores the topological structures of social networks. Thirty-five years after it was proposed, this problem was solved with the development of complex network theory. In 2001, Zanette [11, 12] first applied complex network theory to research on rumor spreading in a small-world network proposed by Watts and Strogatz [13] in 1998. For a more general scenario, Moreno et al. [14] established a rumor-spreading model in 2004 and analyzed the results of a simulation of a scale-free network proposed by Barabási and Albert [15]. On the basis of this work, a number of scholars have proposed adjustments to the classic model. Wang et al. [16] proposed that the ignorant (people who have never heard of the rumor) in the SIRaRu model will refuse to believe the rumor with a certain probability after hearing it. In the same year, Wang et al. [17] considered the existence of two rumors in the same network and proposed the 2 SI2R model, and Zhao et al. $[18,19]$ proposed the SIHR model with mechanisms for forgetting and remembering in homogeneous and heterogeneous networks, respectively.

There are many reasons and factors for why rumors can spread widely [20], including their degree of indiscernibility and the behavior of the individual. Huo and Cheng [21] introduced the degree of indiscernibility of the rumor to the IWSR model and quantitatively described it. In this model, the author describes indiscernible degree of events as a parameter $m$ and then uses the function $f(m)$ to describe transfer rate that ignorant accept and spread rumor. Xia et al. [22] proposed the SEIR model for homogeneous and heterogeneous networks with a hesitation mechanism. In this model, Xia defines the degree of fuzziness of the rumor as the parameter $m$ which conforms to the human mind that people like to explore the truth. The results of their model showed that reducing the fuzziness of events can reduce the impact of rumors. It has been shown that improving the behavior of individuals can make them better reveal a rumor and reduce its impact $[23,24]$. Considering mechanisms for the loss of interest and immunity, Huo et al. [25] proposed the SIbInIu model that divides a given population into four categories and assumed that believers spontaneously lose interest of the rumor with probability $\omega$ . It is concluded that the loss of the mechanisms of interest and immunity have a negative impact on the scale of the final spreading of rumors. In social networks, different people may have different views of a rumor. Zan et al. [7] introduced mechanisms for a counterattack and self-resistance to the SICR model, which means that "counterattack" individuals will persuade "infective" people (people who spread the rumor) to stop spreading the rumor. The degree of indiscernibility of rumors and the behavior of individuals play an important role in the process of their spreading.

Some scholars consider the impact of the antirumor on the spread of the rumor. Unlike most researches on the spreading process of the rumor using epidemic models, Askarizadeh [26] proposed a method based on game theory to study the spreading process of the rumor. This model considers a rumor control mechanism, which is to send antirumor information through the rumor control center, and studies the factors that affect people's decision-making, including social anxiety, people's attitude toward rumor/ antirumor, strength of rumor/antirumor, influence of rumor control centers, and participation of people in discussions. Through simulation, it is concluded that a persuasive antirumor and rumor control center can significantly affect the spread of the rumor. Zhang [27] improved the SIR model, taking into account 8 factors such as information attraction, objective identification of rumors, subjective identification of people, the degree of trust of information media, spread probability, reinforcement coefficient, block value, and expert effects. In this paper, the people are divided into 8 categories, and the influence of various factors on the spread of the rumor is studied by means of simulation. It is concluded that higher subjective and objective identification will prevent rumor spreading. On the basis of the SIR model, Ji [28] considered the two spreading mechanisms of the time when the antirumor joined the network and studied the spreading process of the antirumor on complex networks. Through the simulation method, the relevant conclusions about the time threshold for the antirumor to join the network are drawn: the time threshold is a quantity related to the network structure; it decreases with the increase of the average degree until it decreases to 0 ; the earlier the joining time, the rumor can be controlled as soon as possible; when the antirumor exceeds the time threshold, the time effect will not be able to control the rumor. Jiang [29] separately modeled the two processes of rumor generation and the addition of "anti-rumor" information. Through the parameter sensitivity analysis, the critical point of whether to join the antirumor intervention is obtained; through the sensitivity analysis of the joining time, it is concluded that after the rumors appear, the officials will take actions as soon as possible to reduce the loss and impact caused by the rumor. The above several articles have considered the role of "antirumors," and all adopt simulation methods to conduct steady-state analysis of their respective models and draw relevant conclusions, but the focus is different. Askarizadeh uses the idea of game theory to build the model, while the other three articles are model improvements based on the 
infectious disease model. Comparing the Ji and Jiang, the others focus more on the research on the factors that affect the spread of rumors, so as to find out how the factors affect the spread of rumors, while Ji and Jiang more focused on researching the time when "anti-rumors" joined the network.

Due to the confusing nature of rumors and the differences in individual behaviors, rumors can be widely spread in social networks. The abovementioned literature more or less considered the above two elements and established a spreading dynamic model to study the phenomenon of rumor spreading, but most articles did not consider these two elements at the same time. Secondly, only one type of spreader has been considered (the person who spreads the rumor) in most spreading models. In practice, when a major rumor is spread, it is identified by authoritative media or the government, which then spreads the truth. Thirdly, in prevalent research is that a number of models have low versatility on the model topology, which means that they are rendered inapplicable such that they cannot be adjusted if certain conditions change. And most scholars believe that after interacting with rumor spreader, the ignorant will turn into spreader or exposed (SEIR) with series topology in SIR spreading model or SEIR spreading model separately.

Therefore, we use past research to build a 2 SIH2R (spreader1-spreader2-ignorant-hesitant1-stiflers1-stiflers2) model with parallel topology, discernibility mechanism, and confrontation mechanism in a heterogeneous network. People in this model are divided into six categories and two kinds of spreaders are simultaneously considered (people who spread the rumor and those who spread the truth). This 2SIH2R model can be converted into the traditional SIR or the SEIR model by changing the relevant parameters.

To sum up, in theory, the 2SIH2R model is compatible with the basic assumptions of the traditional model and incorporates more realistic elements. From a perspective, our model is more convincing. For rumor control in real life, this model can quantitatively study how the discernible and confrontation mechanisms affect the spread of rumors. So, the government can take corresponding realistic measures to adjust the discernible and confrontation mechanisms to achieve the goal of information governance.

The remainder of this paper is organized as follows: In Section 2, we define the 2SIH2R model and establish its mean-field equations. In Section 3, a steady-state analysis and the spreading threshold are discussed in three cases. Section 4 details a numerical simulation to test the proposed 2SIH2R model on a heterogeneous network, and Section 5 offers a discussion of the results and summarizes the conclusions of this study.

\section{2SIH2R Rumor-Spreading Model}

In this section, we assume that there are two kinds of information in the entire population, called rumor and truth, and that people in the heterogeneous network can be divided into six categories: spreader1 $\left(S_{1}\right)$, spreader2 $\left(S_{2}\right)$, ignorant $(I)$, hesitant1 $(H)$, stiflers $1\left(R_{1}\right)$, and stiflers $2\left(R_{2}\right)$. Of them, spreader1 and spreader2 represent people who spread the rumor and the truth, respectively. The ignorant are people who have never heard of the rumor or the truth. The term "hesitant1" represents people who have heard the rumor, but do not spread it temporarily, and stiflers1 and stiflers2, respectively, represent people who have heard the rumor and the truth but do not spread them. Referring to the previous research [30], the rumor-spreading process of the $2 \mathrm{SIH} 2 \mathrm{R}$ is shown in Figure 1.

In Figure 1, the solid/dashed line originating from the "ignorant" shows that the ignorant comes into contact with spreader1/spreader2, and the rules of rumor spreading of the $2 \mathrm{SIH} 2 \mathrm{R}$ model can be summarized as follows:

(1) From the perspective of equation rumors $=i \times a$ and reality, considering that different rumor events have different confusion, which takes impact to the people is also different, so, we define $m$ as the rate of discernibility of the rumor and $f(m)$ as the ability of people to reveal it. The function of $f$ is to map the characteristics of the rumor to those of the people. We assume that there is a positive correlation between $m$ and $f(m)$. The greater $m$ is, the more likely the rumor is to be revealed, and the greater the value of $f(m)$ is, the more likely people are to not immediately believe the rumor

(2) When an ignorant person comes into contact with a spreader1, there are three possible scenarios: (i) the ignorant is likely to believe the rumor and spread it with probability $(1-f(m)) \lambda_{1}$, where $\lambda_{1}$ is the rate of spread of the rumor. (ii) The ignorant does not believe the rumor immediately and hesitates to spread it with a probability $f(m) \eta$, where $\eta$ is the potential rate of spreading of the rumor. (iii) The ignorant makes no response to the rumor owing to a lack of interest in it and continues being unaware of it

(3) When an ignorant comes into contact with a spreader2, there are two possible scenarios: (i) The ignorant is likely to believe the truth and spread it with a probability $\lambda_{2}$, called the truth-spreading rate. (ii) The ignorant may make no response to the truth owing to a lack of interest in the truth and continue to be unaware. Because the truth is generally released by an authority or government, it is relatively more objective, accurate, and clear. This makes it easier for the ignorant to judge it, which in turn renders them less likely to be hesitant to spread it

(4) People regarded as hesitant1 have a desire to spread information. Based on suspicion of the rumor and the influence of external circumstances, they do not spread it over a short period. In the process of hesitating, hesitant 1 may choose to believe and spread the rumor, or they may find out and spread the truth. Hence, we suppose that in each step, hesitantl spontaneously transforms into a spreader of information with a certain probability, that is, becomes spreader 1 or spreader 2 with probability $\theta_{1}$ or $\theta_{2}$, respectively

(5) When a spreader1 (spreader2) comes into contact with another spreader1 (spreader2), the exchange of information leads them to believe that the rumor (truth) has been widely known. Thus, spreader1 


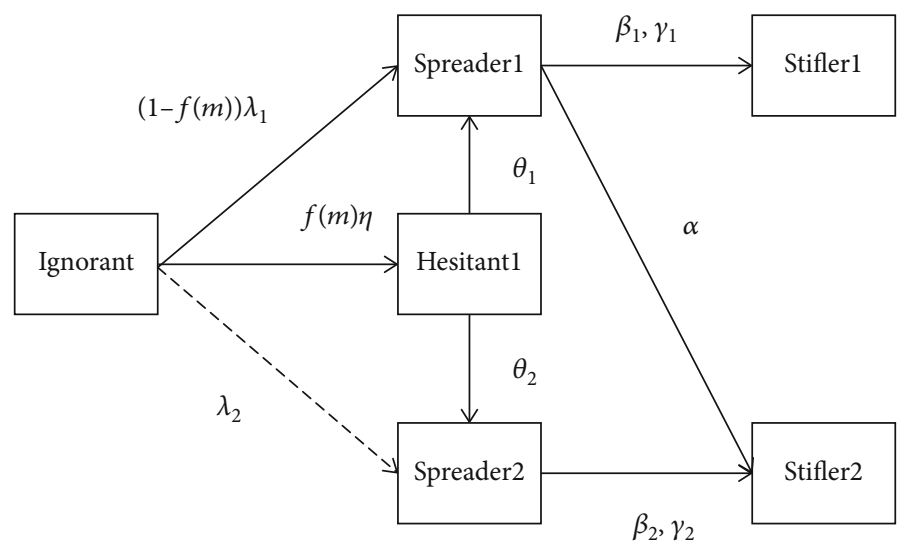

FIGURE 1: 2SIH2R rumor-spreading process.

(spreader2) loses enthusiasm for continually spreading the rumor (truth) and becomes a stifler1 (stifler2), with a rate of loss of interest of $\beta_{1}\left(\beta_{2}\right)$.

(6) Considering the information forgetting mechanism, in each step, spreader1 (spreader2) spontaneously transforms into stifler1 (stifler2) with probability $\gamma_{1}$ $\left(\gamma_{2}\right)$, which means the rumor- (truth-) forgetting rate. This is because people may forget the rumor after a certain period

(7) Considering the mechanism of confrontation between the truth and the rumor, when a spreader1 comes into contact with a spreader2, the former believes the truth rather than the rumor with probability $\alpha$, which is the called the rate of confrontation

In a heterogeneous network, $I_{k}(t), S_{1 k}(t), S_{2 k}(t), H_{k}(t)$, $R_{1 k}(t)$, and $R_{2 k}(t)$ represent the densities of the ignorant, spreader1, spreader2, hesitant1, stifler1, and stifler2 with degree $k$, respectively, at time $t$. Based on the above rules of spreading of the $2 \mathrm{SIH} 2 \mathrm{R}$ model, the mean-field equations can be given as follows:

$$
\begin{aligned}
\frac{d I_{k}(t)}{d t}=- & {\left[\lambda_{1}(1-f(m))+f(m) \eta\right] k I_{k}(t) \sum_{k^{\prime}} S_{1 k^{\prime}}(t) P\left(k^{\prime} / k\right) } \\
& -\lambda_{2} k I_{k}(t) \sum_{k^{\prime}} S_{2 K^{\prime}}(t) P\left(k^{\prime} / k\right),
\end{aligned}
$$

$$
\begin{aligned}
\frac{d S_{1 k}(t)}{d t}= & \lambda_{1}(1-f(m)) k I_{k}(t) \sum_{k^{\prime}} S_{1 k^{\prime}}(t) P\left(k^{\prime} / k\right) \\
& -\alpha k S_{1 k}(t) \sum_{k^{\prime}} S_{2 k^{\prime}}(t) P\left(k^{\prime} / k\right)-\beta_{1} k S_{1 k}(t) \sum_{k^{\prime}}\left(S_{1 k^{\prime}}(t)\right. \\
& \left.+R_{1 k^{\prime}}(t)+H_{k^{\prime}}(t)\right) P\left(k^{\prime} / k\right)-\gamma_{1} S_{1 k}(t)+\theta_{1} H_{k}(t),
\end{aligned}
$$

$$
\begin{aligned}
\frac{d S_{2 k}(t)}{d t}= & \lambda_{2} k I_{k}(t) \sum_{k^{\prime}} S_{2 k^{\prime}}(T) P\left(k^{\prime} / k\right)-\beta_{2} k S_{2 k}(t) \sum_{k^{\prime}}\left(S_{2 k^{\prime}}(t)\right. \\
& \left.+R_{2 k^{\prime}}(t)\right) P\left(k^{\prime} / k\right)-\gamma_{2} S_{2 k}(t)+\theta_{2} H_{k}(t) \\
\frac{d H_{k}(t)}{d t}= & f(m) \eta k I_{k}(t) \sum_{k^{\prime}} S_{1 k^{\prime}}(t) P\left(k^{\prime} / k\right)-\left(\theta_{1}+\theta_{2}\right) H_{k}(t), \\
& \frac{d R_{1 k}(t)}{d t}=\beta_{1} k S_{1 k}(t) \sum_{k^{\prime}}\left(S_{1 k^{\prime}}(t)+R_{1 k^{\prime}}(t)\right. \\
& \left.+H_{k^{\prime}}(t)\right) P\left(k^{\prime} / k\right)+\gamma_{1} S_{1 k}(t), \\
\frac{d R_{2 k}(t)}{d t}= & \alpha k S_{1 k}(t) \sum_{k^{\prime}} S_{2 k^{\prime}}(T) P\left(k^{\prime} / k\right)+\beta_{2} k S_{2 k}(t) \sum_{k^{\prime}}\left(S_{2 k^{\prime}}(t)\right. \\
& \left.+R_{2 k^{\prime}}(t)\right) P\left(k^{\prime} / k\right)+\gamma_{2} S_{2 k}(t),
\end{aligned}
$$

where $I_{k}=N_{I, K} / N_{k}$ represents the number of the ignorant with degree $k$ divided by the total number of people with degree $k, P\left(k^{\prime} / k\right)$ is the probability that a node with degree $k$ is linked to one with degree $k^{\prime}$, and $\sum_{k^{\prime}} S_{1 k^{\prime}}(t) P\left(k^{\prime} / k\right)$ is the probability that an edge of a node with degree $k$ links to a spreader 1 node at time $t$.

\section{Steady-State Analysis}

In this section, we provide the condition whereby rumors and the truth can spread successfully in a heterogeneous network. It is assumed that when the model arrives at a steady state, neither spreader1 nor spreader2 is present. We can thus obtain the conclusions $S_{1}=\lim _{t \longrightarrow \infty} S_{1}(t)=0, S_{2}=\lim _{t \rightarrow \infty} S_{2}(t)=0, H$ $=\lim _{t \rightarrow \infty} H(t)=0$, and $\lim _{t \rightarrow \infty}\left(I(t)+R_{1}(t)+R_{2}(t)\right)=1$. Moreover, the normalization condition is $I(t)+S_{1}(t)+S_{2}(t)$ $+H(t)+R_{1}(t)+R_{2}(t)=1$. The degree-degree correlations can be described as $P\left(k^{\prime} / k\right)=k^{\prime} P\left(k^{\prime}\right) /<k>=q\left(k^{\prime}\right)$, where 
$P\left(k^{\prime}\right)$ is the degree distribution function. Without loss of generality, we set the density of the ignorant to $I_{k}(0)=I(0)$ $\approx 1$. We now study the spreading threshold of the model in three cases.

3.1. Steady-State Analysis of Rumor. In the early stages of its process of spreading, few people know the rumor and no one knows the truth. Thus, in this case, without loss of generality, we assume that there is only one spreader1 who spreads the rumor, and there is no truth. It is assumed that there are $N$ nodes in the network. Based on the above, the initial condition can be given as follows: $S_{2}(0)=0, S_{1}(0)=1 / N \approx 0, I($ $0)=N-1 / N \approx 1$, and $H(0)=R_{1}(0)=R_{2}(0)=0$, and the model can be adjusted as follows:

$$
\frac{d I_{k}(t)}{d t}=-\left[\lambda_{1}(1-f(m))+f(m) \eta\right] k I_{k}(t) \sum_{k^{\prime}} S_{1 k^{\prime}}(t) q\left(k^{\prime}\right)
$$

$$
\begin{aligned}
\frac{d S_{1 k}(t)}{d t}= & \lambda_{1}(1-f(m)) k I_{k}(t) \sum_{k^{\prime}} S_{1 k^{\prime}}(t) q\left(k^{\prime}\right) \\
& -\beta_{1} k S_{1 k}(t) \sum_{k^{\prime}}\left(S_{1 k^{\prime}}(t)+R_{1 k^{\prime}}(t)+H_{k^{\prime}}(t)\right) q\left(k^{\prime}\right) \\
& -\gamma_{1} S_{1 k}(t)+\theta_{1} H_{k}(t),
\end{aligned}
$$

$$
\begin{gathered}
\frac{d H_{k}(t)}{d t}=f(m) \eta k I_{k}(t) \sum_{k^{\prime}} S_{1 k^{\prime}}(t) q\left(k^{\prime}\right)-\theta_{1} H_{k}(t), \\
\frac{d R_{1 k}(t)}{d t}=\beta_{1} k S_{1 k}(t) \sum_{k^{\prime}}\left(S_{1 k^{\prime}}(t)+R_{1 k^{\prime}}(t)+H_{k^{\prime}}(t)\right) q\left(k^{\prime}\right)+\gamma_{1} S_{1 k}(t) .
\end{gathered}
$$

In this case, we can integrate Eq. (7) directly and get

$$
I_{k}(t)=e^{-\left(\lambda_{1}(1-f(m))+f(m) \eta\right) k \varphi(t)}
$$

where $\varphi(t)$ can be given as

$$
\varphi(t)=\int_{0}^{t} \sum_{k^{\prime}} S_{1 k^{\prime}}\left(t^{\prime}\right) q\left(k^{\prime}\right) d t^{\prime}=\int_{0}^{t}<<S_{1 k^{\prime}}\left(t^{\prime}\right)>>d t^{\prime},
$$

and the shorthand notation $<<O(k)>>=\sum_{k} O(k) q(k)$ has been used.

Multiplying Eq. (8) with $q(k)$, summing over $k$, and integrating the equation with respect to $t$, the following deriva- tion can be obtained:

$$
\begin{aligned}
\frac{d \varphi(t)}{d t}= & \lambda_{1}(1-f(m)) \sum_{k} q(k) \int_{0}^{t} k e^{-\left(\lambda_{1}(1-f(m))+f(m) \eta\right) k \varphi\left(t^{\prime}\right)} \\
<< & S_{1 k^{\prime}}\left(t^{\prime}\right)>>d t^{\prime}-\beta_{1} \int_{0}^{t} \sum_{k} k S_{1 k}\left(t^{\prime}\right) q(k) \sum_{k^{\prime}}\left(S_{1 k^{\prime}}\left(t^{\prime}\right)\right. \\
& \left.+R_{1 k^{\prime}}\left(t^{\prime}\right)+H_{k^{\prime}}\left(t^{\prime}\right)\right) q\left(k^{\prime}\right) d t^{\prime} \\
& -\gamma_{1} \int_{0}^{t} \sum_{k} S_{1 k}\left(t^{\prime}\right) q(k) d t^{\prime}+\theta_{1} \int_{0}^{t} \sum_{k} H_{k}\left(t^{\prime}\right) q(k) d t^{\prime} \\
= & \frac{\lambda_{1}(1-f(m))}{\lambda_{1}(1-f(m))+f(m) \eta}\left[1-<<e^{-\left(\lambda_{1}(1-f(m))+f(m) \eta\right) k \varphi(t)}>>\right] \\
& -\gamma_{1} \varphi(t)+\theta_{1} \psi(t)-\beta_{1} \int_{0}^{t} \\
< & <k S_{1 k}\left(t^{\prime}\right)>>\left[1-<<e^{-\left(\lambda_{1}(1-f(m))+f(m) \eta\right) k \varphi\left(t^{\prime}\right)}>>\right] d t^{\prime},
\end{aligned}
$$

where $\psi(t)=\int_{0}^{t}<<H_{k}\left(t^{\prime}\right)>>d t^{\prime}$.

Similarly, by multiplying Eq. (9) with $q(k)$, summing over $k$, and integrating the equation, we can get

$$
\frac{d \psi(t)}{d t}=\frac{f(m) \eta}{\lambda_{1}(1-f(m))+f(m) \eta}\left[1-<<e^{-\left(\lambda_{1}(1-f(m))+f(m) \eta\right) k \varphi(t)}>>\right]-\theta_{1} \psi(t) .
$$

When $t \longrightarrow \infty, d \varphi / d t=0$, and $d \psi / d t=0$, the following derivation can be obtained:

$$
\begin{aligned}
0= & \frac{\lambda_{1}(1-f(m))}{\lambda_{1}(1-f(m))+f(m) \eta}\left[1-<<e^{-\left(\lambda_{1}(1-f(m))+f(m) \eta\right) k \varphi_{\infty}}>>\right] \\
& -\gamma_{1} \varphi_{\infty}+\theta_{1} \psi(\infty)-\beta_{1} \int_{0}^{\infty}<<k S_{1 k}\left(t^{\prime}\right)>> \\
& \cdot\left[1-<<e^{-\left(\lambda_{1}(1-f(m))+f(m) \eta\right) k \varphi\left(t^{\prime}\right)}>>\right] d t^{\prime},
\end{aligned}
$$

$$
\begin{aligned}
0= & \frac{f(m) \eta}{\lambda_{1}(1-f(m))+f(m) \eta}\left[1-<<e^{-\left(\lambda_{1}(1-f(m))+f(m) \eta\right) k \varphi_{\infty}}>>\right] \\
& -\theta_{1} \psi(\infty),
\end{aligned}
$$

where $\varphi_{\infty}=\varphi(\infty)$.

Combining Eqs. (15) and (16),

$$
\begin{aligned}
0= & {\left[1-<<e^{-\left(\lambda_{1}(1-f(m))+f(m) \eta\right) k \varphi_{\infty}>>}\right] } \\
& -\beta_{1} \int_{0}^{\infty}<<k S_{1 k}\left(t^{\prime}\right)>>\left[1-<<e^{-\left(\lambda_{1}(1-f(m))+f(m) \eta\right) k \varphi\left(t^{\prime}\right)}>>\right] d t^{\prime} \\
& -\gamma_{1} \varphi_{\infty} .
\end{aligned}
$$


By integrating Eqs. (8) and (9), we have

$$
\begin{aligned}
S_{1 k}(t)= & \frac{\lambda_{1}(1-f(m))}{\lambda_{1}(1-f(m))+f(m) \eta}\left(1-e^{-\left(\lambda_{1}(1-f(m))+f(m) \eta\right) k \varphi(t)}\right) \\
& -\gamma_{1} \int_{0}^{t} S_{1 k}\left(t^{\prime}\right) d t^{\prime}+\theta_{1} \int_{0}^{t} H_{k}\left(t^{\prime}\right) d t^{\prime} \\
& -\beta_{1} k \int_{0}^{t} S_{1 k}\left(t^{\prime}\right)\left(1-<<e^{-\left(\lambda_{1}(1-f(m))+f(m) \eta\right) k \varphi(t)}>>\right) d t^{\prime}, \\
H_{k}(t)= & \frac{f(m) \eta}{\lambda_{1}(1-f(m))+f(m) \eta}\left(1-e^{-\left(\lambda_{1}(1-f(m))+f(m) \eta\right) k \varphi(t)}\right) \\
& -\theta_{1} \int_{0}^{t} H_{k}\left(t^{\prime}\right) d t^{\prime} .
\end{aligned}
$$

By adding Eq. (18) to Eq. (19), we have

$$
\begin{aligned}
S_{1 k}(t)= & 1-e^{-\left(\lambda_{1}(1-f(m))+f(m) \eta\right) k \varphi(t)}-H_{k}(t) \\
& -\gamma_{1} \int_{0}^{t} S_{1 k}\left(t^{\prime}\right) d t^{\prime}-\beta_{1} k \int_{0}^{t} S_{1 k}\left(t^{\prime}\right) \\
& \cdot\left(1-\ll e^{-\left(\lambda_{1}(1-f(m))+f(m) \eta\right) k \varphi(t)} \gg\right) d t^{\prime} .
\end{aligned}
$$

By solving Eq. (19), we can obtain

$$
\begin{aligned}
H_{k}(t)= & \frac{f(m) \eta}{\lambda_{1}(1-f(m))+f(m) \eta} \\
& \cdot\left[1-e^{-\left(\lambda_{1}(1-f(m))+f(m) \eta\right) k \varphi(t)}\right. \\
& \left.-\theta_{1} \int_{0}^{t}\left(1-e^{-\left(\lambda_{1}(1-f(m))+f(m) \eta\right) k \varphi\left(t^{\prime}\right)}\right) e^{\theta_{1}\left(t^{\prime}-t\right)} d t^{\prime}\right] .
\end{aligned}
$$

By inserting Eq. (21) into Eq. (20), we can get

$$
\begin{aligned}
S_{1 k}(t)= & \frac{\lambda_{1}(1-f(m))}{\lambda_{1}(1-f(m))+f(m) \eta}\left(1-e^{-\left(\lambda_{1}(1-f(m))+f(m) \eta\right) k \varphi(t)}\right) \\
& +O\left(\beta_{1}\right)-\gamma_{1} \int_{0}^{t}\left[\frac{\lambda_{1}(1-f(m))}{\lambda_{1}(1-f(m))+f(m) \eta}\right. \\
& \cdot\left(1-e^{-\left(\lambda_{1}(1-f(m))+f(m) \eta\right) k \varphi\left(t^{\prime}\right)}\right) e^{\gamma_{1}\left(t^{\prime}-t\right)} d t^{\prime} \\
& -\gamma_{1} \int_{0}^{t} \theta_{1}\left[\int_{0}^{t^{\prime}} \frac{f(m) \eta}{\lambda_{1}(1-f(m))+f(m) \eta}\right. \\
& \left.\cdot\left(1-e^{-\left(\lambda_{1}(1-f(m))+f(m) \eta\right) k \varphi\left(t^{\prime \prime}\right)}\right) e^{\theta_{1}\left(t^{\prime \prime}-t^{\prime}\right)} d t^{\prime \prime}\right] e^{\gamma_{1}\left(t^{\prime}-t\right)} d t^{\prime} . \\
& +\frac{f(m) \eta}{\lambda_{1}(1-f(m))+f(m) \eta} \theta_{1}^{t} \int_{0}^{t} \\
& \cdot\left(1-e^{\left.-\left(\lambda_{1}(1-f(m))+f(m) \eta\right)\right) k \varphi\left(t^{\prime}\right)}\right) e^{\theta_{1}\left(t^{\prime}-t\right)} d t^{\prime}
\end{aligned}
$$

Both $\varphi(t)$ and $\varphi_{\infty}$ are very small when the size of the rumor tends to be stable. There is thus a finite function $g(t$
) that satisfies $\varphi(t)=\varphi_{\infty} g(t)$, and Eq. (22) then becomes

$$
\begin{aligned}
S_{1 k}(t)= & k \varphi_{\infty}\left\{\lambda_{1}(1-f(m)) g(t)-\gamma_{1} \lambda_{1}(1-f(m)) \int_{0}^{t} g\left(t^{\prime}\right) e^{\gamma_{1}\left(t^{\prime}-t\right)} d t^{\prime}\right. \\
& +O\left(\beta_{1}\right)-\gamma_{1} \theta_{1} f(m) \eta \int_{0}^{t} e^{\gamma_{1}\left(t^{\prime}-t\right)} \int_{0}^{t^{\prime}} g\left(t^{\prime \prime}\right) e^{\theta_{1}\left(t^{\prime \prime}-t^{\prime}\right) d t^{\prime \prime} d t^{\prime}} \\
& \left.+f(m) \eta \theta_{1} \int_{0}^{t} g\left(t^{\prime}\right) e^{\theta_{1}\left(t^{\prime}-t\right)} d t^{\prime}+O\left(\varphi_{\infty}^{2}\right)\right\} .
\end{aligned}
$$

Inserting Eq. (23) into Eq. (17),

$$
\begin{aligned}
& 0=1-<<1-\left(\lambda_{1}(1-f(m))+f(m) \eta\right) k \varphi_{\infty} \\
& +\frac{\left[\left(\lambda_{1}(1-f(m))+f(m) \eta\right) k\right]^{2} \varphi_{\infty}^{2}}{2}+O\left(\varphi_{\infty}^{3}\right)>> \\
& -\beta_{1} \int_{0}^{\infty}<<k \varphi_{\infty} k\left\{\lambda_{1}(1-f(m)) g\left(t^{\prime}\right)\right. \\
& -\gamma_{1} \lambda_{1}(1-f(m)) \int_{0}^{t^{\prime}} g\left(t^{\prime \prime}\right) e^{\gamma_{1}\left(t^{\prime \prime}-t^{\prime}\right)} d t^{\prime \prime} \\
& +f(m) \eta \theta_{1} \int_{0}^{t^{\prime}} g\left(t^{\prime \prime}\right) e^{\theta_{1}\left(t^{\prime \prime}-t^{\prime}\right)} d t^{\prime \prime} \\
& \left.-\gamma_{1} \theta_{1} f(m) \eta \int_{0}^{t^{\prime}} e^{\gamma_{1}\left(t^{\prime \prime}-t^{\prime}\right)} \int_{0}^{t^{\prime}} g\left(t^{\prime \prime \prime}\right) e^{\theta_{1}\left(t^{\prime \prime \prime}-t^{\prime \prime}\right)} d t^{\prime \prime \prime} d t^{\prime \prime}\right\} \\
& +O\left(\varphi_{\infty}^{2}\right)+O\left(\beta_{1}\right)>> \\
& \times\left[1-<<1-\left(\lambda_{1}(1-f(m))+f(m) \eta\right) k \varphi_{\infty} g\left(t^{\prime}\right)+O\left(\varphi_{\infty}^{2}\right)>>\right] d t^{\prime} \\
& -\gamma_{1} \varphi_{\infty} \text {, } \\
& 0=\varphi_{\infty}\left\{\left[\lambda_{1}(1-f(m))+f(m) \eta\right]<<k>>\right. \\
& -\frac{\left(\lambda_{1}(1-f(m))+f(m) \eta\right)^{2}}{2}<<k^{2}>>\varphi_{\infty} \\
& \left.-\beta_{1}<<k^{2}>>I\left(\lambda_{1}(1-f(m))+f(m) \eta\right)<<k>>\varphi_{\infty}-\gamma_{1}\right\} \\
& +O\left(\varphi_{\infty}^{3}\right)+O\left(\beta_{1}^{2}\right) \text {. }
\end{aligned}
$$

where $I=\int_{0}^{\infty}\left\{\lambda_{1}(1-f(m)) g\left(t^{\prime}\right)-\gamma_{1} \lambda_{1}(1-f(m)) \int_{0}^{t^{\prime}} g\left(t^{\prime \prime}\right)\right.$ $e^{\gamma_{1}\left(t^{\prime \prime}-t^{\prime}\right)} d t^{\prime \prime}+f(m) \eta \theta_{1} \int_{0}^{t^{\prime}} g\left(t^{\prime \prime}\right) e^{\theta_{1}\left(t^{\prime \prime}-t^{\prime}\right)} d t^{\prime \prime}-\gamma_{1} \theta_{1} f(m) \eta \int_{0}^{t^{\prime}}$ $\left.e^{\gamma_{1}\left(t^{\prime \prime}-t^{\prime}\right)} \int_{0}^{t^{\prime}} g\left(t^{\prime \prime \prime}\right) e^{\theta_{1}\left(t^{\prime \prime}-t^{\prime \prime}\right)} d t^{\prime \prime \prime} d t^{\prime \prime}\right\} g\left(t^{\prime}\right) d t^{\prime}$, which is a finite and positive-definite integral.

From Eq. (25), we have

$$
\begin{aligned}
0= & \varphi_{\infty}\left\{\left[\lambda_{1}(1-f(m))+f(m) \eta\right]<<k>>\right. \\
& -\frac{\left(\lambda_{1}(1-f(m))+f(m) \eta\right)^{2}}{2}<<k^{2}>>\varphi_{\infty} \\
& \left.-\beta_{1}<<k^{2}>><<k>>I\left(\lambda_{1}(1-f(m))+f(m) \eta\right) \varphi_{\infty}-\gamma_{1}\right\} .
\end{aligned}
$$

$\varphi_{\infty}=0$ is one solution to Eq. (26), and the nontrivial 
solution is obtained as follows:

$\varphi_{\infty}=\frac{\left[\lambda_{1}(1-f(m))+f(m) \eta\right]<<k>>-\gamma_{1}}{<<k^{2}>>\left[\lambda_{1}(1-f(m))+f(m) \eta\right]\left\{1 / 2\left[\lambda_{1}(1-f(m))+f(m) \eta\right]+\beta_{1}<<k>>I\right\}}$.

Note that $\ll k \gg=\left\langle k^{2}\right\rangle /<k>$; so, $\left[\lambda_{1}(1-f(m))+f(\right.$ $m) \eta]\left(\left\langle k^{2}>\right|<k>\right)-\gamma_{1} \geq 0$, that is, $\lambda_{1} \geq<k>\gamma_{1} /<k^{2}>-f(m$ $\eta \eta(1-f(m))$, which yields a positive value for $\varphi_{\infty}$. This means that the spreading threshold in this case is

$$
\lambda_{1 c}=\frac{\left(<k>/<k^{2}>\right) \gamma_{1}-f(m) \eta}{(1-f(m))} .
$$

3.2. Steady-State Analysis of Truth. In this case, we consider the truth of some well-known events has been already spread by the government or authoritative media. In this period of time, there are no rumors, but only the truth. Based on the above, the initial condition can be given as follows: $S_{1}(0)=$ $0, S_{2}(0)=1 / N \approx 0, I(0)=N-1 / N \approx 1$, and $H(0)=R_{1}(0)=$ $R_{2}(0)=0$. We can prove that in this case, the model can be adjusted as follows:

$$
\begin{gathered}
\frac{d I_{k}(t)}{d t}=-\lambda_{2} k I_{k}(t) \sum_{k^{\prime}} S_{2 k^{\prime}}(t) P\left(k^{\prime} / k\right), \\
\frac{d S_{2 k}(t)}{d t}=\lambda_{2} k I_{k}(t) \sum_{k^{\prime}} S_{2 k^{\prime}}(t) q\left(k^{\prime}\right)-\beta_{2} k S_{2 k}(t) \sum_{k^{\prime}}\left(S_{2 k^{\prime}}(t)\right. \\
\left.+R_{2 k^{\prime}}(t)\right) q\left(k^{\prime}\right)-\gamma_{2} S_{2 k}(t), \\
\frac{d R_{2 k}(t)}{d t}=\beta_{2} k S_{2 k}(t) \sum_{k^{\prime}}\left(S_{2 k^{\prime}}(t)+R_{2 k^{\prime}}(t)\right) P\left(k^{\prime} / k\right)+\gamma_{2} S_{2}(t) .
\end{gathered}
$$
get

We follow the proof procedure in the previous section to

$$
\lambda_{2 c}=\frac{<k>}{<k^{2}>} \gamma_{2}
$$

3.3. Steady-State Analysis of the 2SIH2R Model. In this case, we consider a more general situation where the rumor and the truth exist in the network at the same time. Without loss of generality, we assume that there is one spreader1 and one spreader2 at time $t=0$, and the initial condition can then be given as follows: $I(0)=N-2 / N \approx 1, S_{1}(0)=S_{2}(0)=1 / N \approx 0$, and $H(0)=R_{1}(0)=R_{2}(0)=0$. Without loss of generality, it is assumed that $I_{k}(0)=1, S_{1 k}(0)=S_{2 k}(0) \approx 0$, and $H_{k}(0)=$ $R_{1 k}(0)=R_{2 k}(0)=0$.

For $I_{k}=N_{I, K} / N_{k}$ and $I_{k}(t)+S_{1 k}(t)+S_{2 k}(t)+H_{k}(t)+$ $R_{1 k}(t)+R_{2 k}(t)=1$, i.e., if the number of five types of people is fixed, there is only one corresponding value of the remaining one. So, we will discuss the system (1)-(5). Obviously, the system (1)-(5) has a disease-free equilibrium $E_{0}=\left(I_{k}(0)\right.$, $\left.S_{1 k}(0), S_{2 k}(0), H_{k}(0), R_{1 k}(0), R_{2 k}(0)\right)=(1,0,0,0,0,0)$. The above Jacobin matrix at $E_{0}$ can be written as

$$
J\left(E_{0}\right)=\left[\begin{array}{cccc}
A_{11} & A_{12} & \cdots & A_{1 n} \\
A_{21} & A_{22} & \cdots & A_{2 n} \\
\vdots & \vdots & \ddots & \vdots \\
A_{n 1} & A_{n 2} & \cdots & A_{n n}
\end{array}\right], \text { where }
$$

$$
\begin{aligned}
A_{i j}= & \left(\begin{array}{ccccc}
0 & -\left[\lambda_{1}(1-f(m))+f(m) \eta\right] j q(j) & -\lambda_{2} j q(j) & 0 & 0 \\
0 & \lambda_{1}(1-f(m)) j q(j)-\gamma_{1} & 0 & \theta_{1} & 0 \\
0 & 0 & \lambda_{2} j q(j)-\gamma_{2} & \theta_{2} & 0 \\
0 & f(m) \eta j q(j) & 0 & -\left(\theta_{1}+\theta_{2}\right) & 0 \\
0 & \gamma_{1} & 0 & 0 & 0
\end{array}\right)(i=j), \\
A_{i j} & =\left(\begin{array}{ccccc}
0 & -\left[\lambda_{1}(1-f(m))+f(m) \eta\right] i q(j) & -\lambda_{2} i q(j) & 0 & 0 \\
0 & \lambda_{1}(1-f(m)) i q(j) & 0 & 0 & 0 \\
0 & 0 & \lambda_{2} i q(j) & 0 & 0 \\
0 & f(m) \eta i q(j) & 0 & 0 & 0 \\
0 & 0 & 0 & 0 & 0
\end{array}\right)(i \neq j) .
\end{aligned}
$$

By mathematical induction method, the characteristic equation can be calculated as

$$
\begin{aligned}
0 & \left.=(-\mu)^{2 n}\left(-\gamma_{2}-\mu\right)^{n-1}\left\{\lambda_{2} \sum_{n} n q(n)-\gamma_{2}-\mu\right\}\left[-\left(\theta_{1}+\theta_{2}\right)-\mu\right)\left(-\gamma_{1}-\mu\right)\right]^{n-1} \\
& \times\left\{\left(-\left(\theta_{1}+\theta_{2}\right)-\mu\right)\left[\lambda_{1}(1-f(m)) \sum_{n} n q(n)-\gamma_{1}-\mu\right]-\theta_{1} f(m) \eta \sum_{n} n q(n)\right\} .
\end{aligned}
$$

For $q(k)=k P(k) /<k>$, we have $\sum_{k} k q(k)=\sum_{k} k^{2} P(k) /<k$ $>=\left\langle k^{2}\right\rangle|<k\rangle$. So, the characteristic equation can be rewritten as

$$
\begin{aligned}
0= & (-\mu)^{2 n}\left(-\gamma_{2}-\mu\right)^{n-1}\left\{\lambda_{2} \frac{<k^{2}>}{<k>}-\gamma_{2}-\mu\right\} \\
& \cdot\left[\left(-\left(\theta_{1}+\theta_{2}\right)-\mu\right)\left(-\gamma_{1}-\mu\right)\right]^{n-1}\left\{\mu^{2}+b \mu+c\right\},
\end{aligned}
$$

where

$$
\begin{gathered}
b=-\left[\lambda_{1}(1-f(m)) \frac{<k^{2}>}{<k>}-\gamma_{1}-\left(\theta_{1}+\theta_{2}\right)\right], \\
c=-\left[\lambda_{1}(1-f(m)) \frac{<k^{2}>}{<k>}-\gamma_{1}\right]\left(\theta_{1}+\theta_{2}\right)-\theta_{1} f(m) \eta \frac{<k^{2}>}{<k>} .
\end{gathered}
$$

According to the Routh-Hurwitz criteria, the system is stable at $E_{0}$ only when all eigenvalues are not greater than 0 . Thus, a sufficient condition of $\left(I, S_{1}, S_{2}, H, R_{1}, R_{2}\right)$ to be locally asymptotically stable at $E_{0}$ is $\max \{1 / 2(-b+$ $\left.\left.\sqrt{b^{2}-4 c}\right), \lambda_{2}\left(<k^{2}>\mid<k>\right)-\gamma_{2}\right\} \leq 0$. In other words, if rumors or truth can spread in a heterogeneous network, 
the following conditions need to be satisfied:

$$
\frac{1}{2}\left(-b+\sqrt{b^{2}-4 c}\right)>0 \text { or } \lambda_{2} \frac{<k^{2}>}{<k>}-\gamma_{2}>0 .
$$

Consequently,

$$
\lambda_{1}(1-f(m))+\frac{\theta_{1}}{\theta_{1}+\theta_{2}} f(m) \eta>\frac{<k>}{<k^{2}>} \gamma_{1} \text { or } \lambda_{2}>\frac{<k>}{<k^{2}>} \gamma_{2} .
$$

Thus, if the rumor and the truth can spread widely in the generated network, which is closed and heterogeneous, $\lambda_{1}$ and $\lambda_{2}$ should satisfy Eq. (36). We then set $\lambda_{2}=0, \gamma_{2}=0$, and $\theta_{2}=0$ to get the Eq. (28). By setting $\lambda_{1}=0, f(m)=0$, we can get Eq. (30). We can conclude that the third general case contains the first two special cases.

\section{Numerical Simulation}

In this section, we study important features of the $2 \mathrm{SIH} 2 \mathrm{R}$ model in a heterogeneous network generated through a numerical simulation. By referring to the research [30-34], the following initial conditions can be given: $I(0)=N-2 /$ $N \approx 1, S_{1}(0)=S_{2}(0)=1 / N \approx 0$, and $H(0)=R_{1}(0)=R_{2}(0)=$ 0 . We executed the simulation in the Barabási-Albert scalefree network with $N=100000$, and three edges attach a new node to already existing nodes.

Figure 2 shows the general trends of the density of the six categories (spreader1, spreader2, stifler1, stifler2, ignorant, and hesitant) in the 2SIH2R model over time, with parameters $m=0.3, f(m)=0.7 m, \lambda_{1}=\lambda_{2}=0.7, \eta=0.8, \theta_{1}=0.5$, $\theta_{2}=0.3, \beta_{1}=\beta_{2}=0.3, \gamma_{1}=\gamma_{2}=0.1$, and $\alpha=0.5$. Unless otherwise specified, the above parameters are used in this section. The numerical simulation showed that the number of ignorant people declined sharply until it reached the steady state. At the same time, the densities of spreaderl and spreader 2 reached a peak and then declined. When the system became stable, which means that neither the rumor nor the truth spreads, only ignorant, stifler1, and stifler2 remain in the network.

To illustrate how the mechanism affects the spread of the rumor, we plot in Figures 3-5. Figures 3 and 4 show how spreader 1 and stifler 1 change over time with different values of $m$. Figure 5 shows how the final sizes of stifler 1 and stifler2 change with different values of $f(m)$. Figure 3 shows that the peak of spreader1 decreased with increasing $m$, which means that the stronger the mechanism was, the easier it was to reveal the rumor, and the smaller the impact of the rumor was. The same conclusion can be also obtained from Figure 4 . The final size of stifler1 also decreased with increasing $m$. However, the impact of the rumor decreased with increasing $m$, but the times to peak for both spreader 1 and stifler 1 did not change significantly. Figure 5 shows that the final size of stiflerl decreases with increasing values of $f(m)$, but that of stifler 2 increases. It can be concluded that as $m(f(m))$ increases, the short-term and final impacts of the rumor decrease while those of the truth increase.

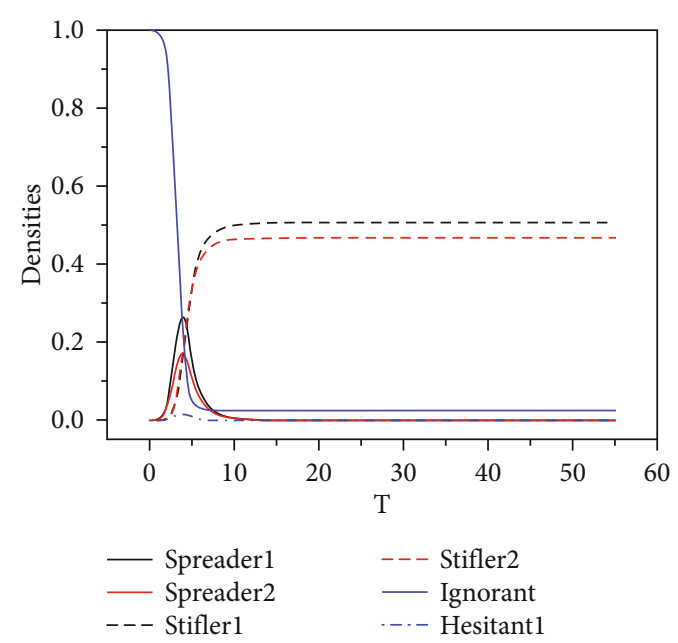

FIgURe 2: Density of six categories of people in the homogeneous network.

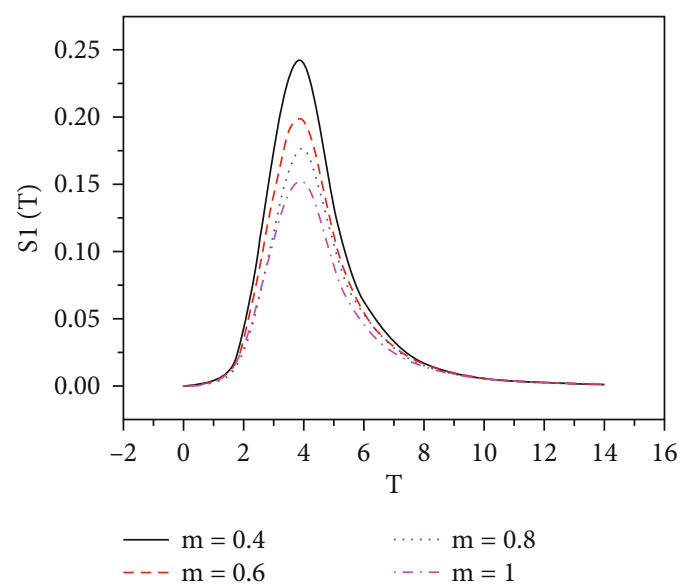

FIgURE 3: Density of spreader1 over time for different values of $m$.

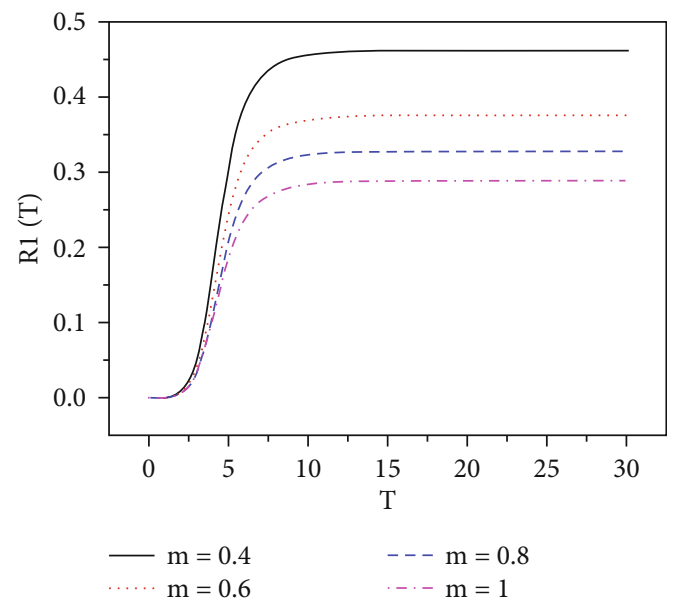

Figure 4: Density of stifler1 over time for different values of $m$.

To illustrate how the confrontation mechanism affected the spread of the rumor, we plot in Figures 6-8. Figures 6 and 7 describe how spreader 1 and stifler 1 change over time with different values of $\alpha$. Figure 8 describes how the final 


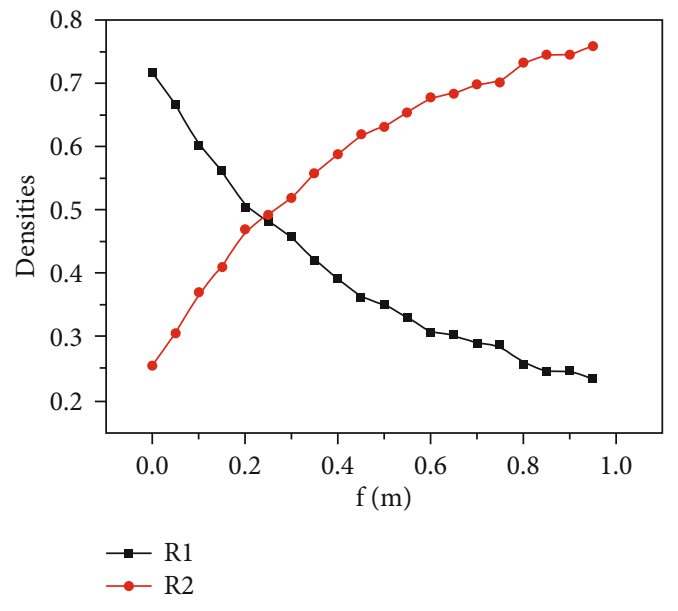

Figure 5: Densities of stifler1 and stifler2 for different values of $f$ $(m)$ in the steady state.

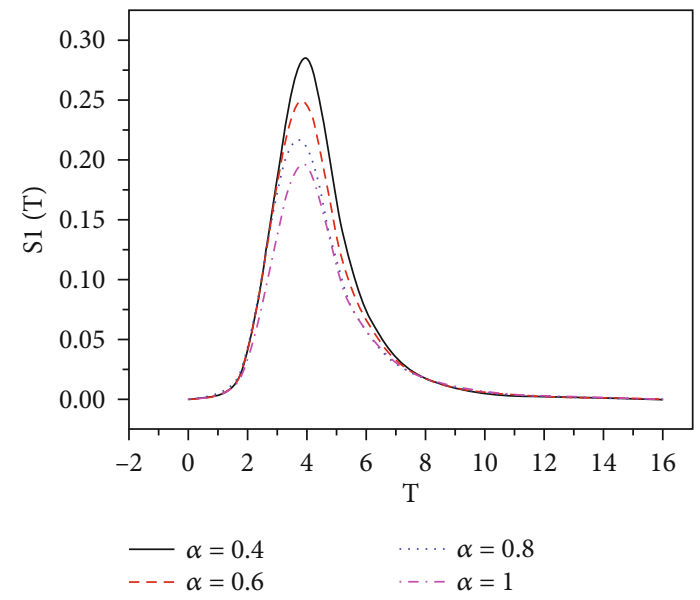

Figure 6: Density of spreader1 over time for different values of $\alpha$.

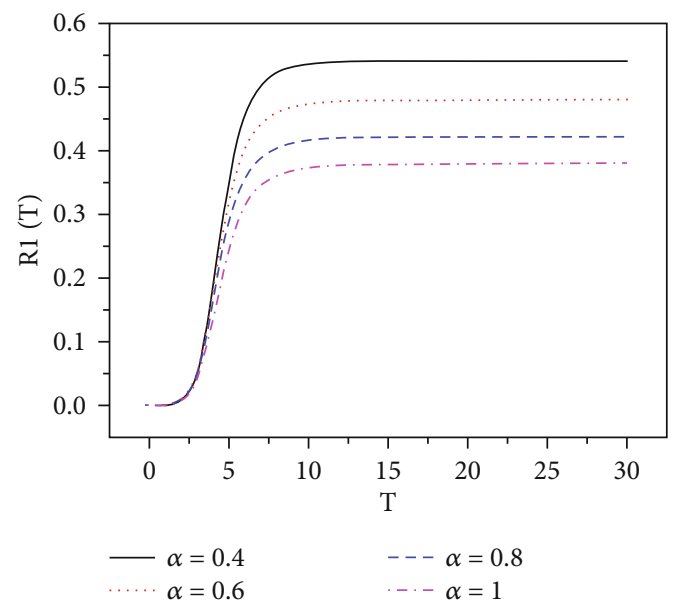

Figure 7: Density of stifler2 over time for different values of $\alpha$.

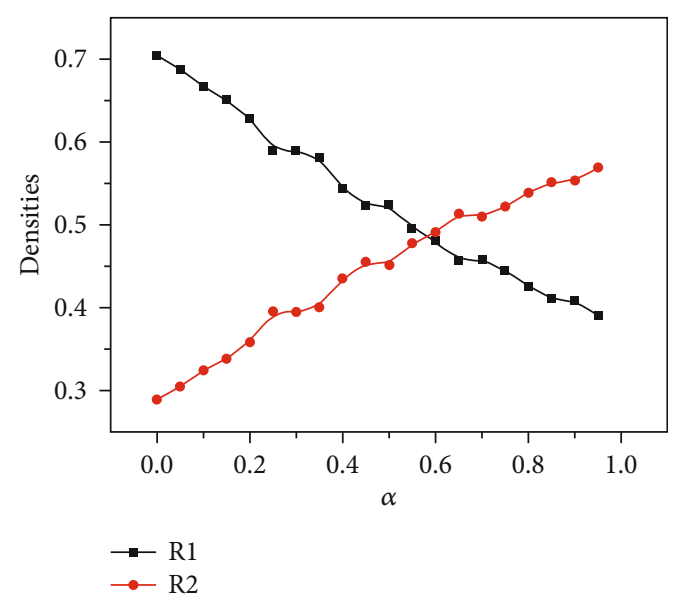

FIgURe 8: Densities of stifler1 and stifler2 for different values of $\alpha$ in the steady-state.

sizes of stifler 1 and stifler 2 change with different values of $\alpha$ .Figure 6 shows that the peak of spreaderl decreased with increasing $\alpha$, which means that the stronger the confrontation mechanism was, the easier it was to reveal the rumor, and the smaller its impact was. The same conclusion can be obtained from Figure 7. The final size of stifler1 also decreased with increasing $\alpha$. However, the impact of the rumor decreased with increasing $\alpha$, but the times to peak of spreader1 and stifler1 did not change significantly. Figure 8 shows that the final size of stifler1 decreases with increasing values of $\alpha$, but that of stifler2 increases. It can be concluded that as $\alpha$ increases, the short-term and final impacts of the rumor will decrease while those of the truth increase.

To illustrate how the mechanisms and $T_{\text {truth_in }}$ affected the spread of the rumor, we plot in Figures 9 and 10. In Figures 9 and 10, the time when the truth begins to spread in the network is called $T_{\text {truth_in }}$, and when $T_{\text {truth in }}=0$, the rumor begins to spread. Figures 9 and 10 describe how the final size of stifler 1 and stifler 2 change over $T_{\text {truth_in }}$ with different values of $f(m)$ and $\alpha$. From Figures 9 and 10, we can find that the earlier the truth joins the network, the influence range of the rumor will be reduced and the truth will be enlarged. As shown in Figure 9, the growth rate of $R_{1}$ and the decline rate of $R_{2}$ both decreased with increasing $T_{\text {truth_in. }}$. In other words, if the truth begins to spread too late, the time factor will not significantly affect the spread of rumors. The same conclusion can also be obtained from Figure 10. Comparing Figures 9 and 10, we can find that if the truth can be spread at an earlier time (E.g., $T_{\text {truth_in }}<5$ ), the influence range of the rumor will be reduced and the truth will be enlarged significantly with increasing $f(m)$ or $\alpha$. But as shown in Figure 10, if the truth begins to spread too late (E.g., $T_{\text {truth_in }}>5$ ), the confrontation mechanism will become ineffective while the discernibility mechanism is still effective.

To illustrate how the mechanisms and $T_{\text {rumor_in }}$ affected the spread of the rumor, we plot in Figures 11 and 12. In Figures 11 and 12, the time when the rumor begins to spread 


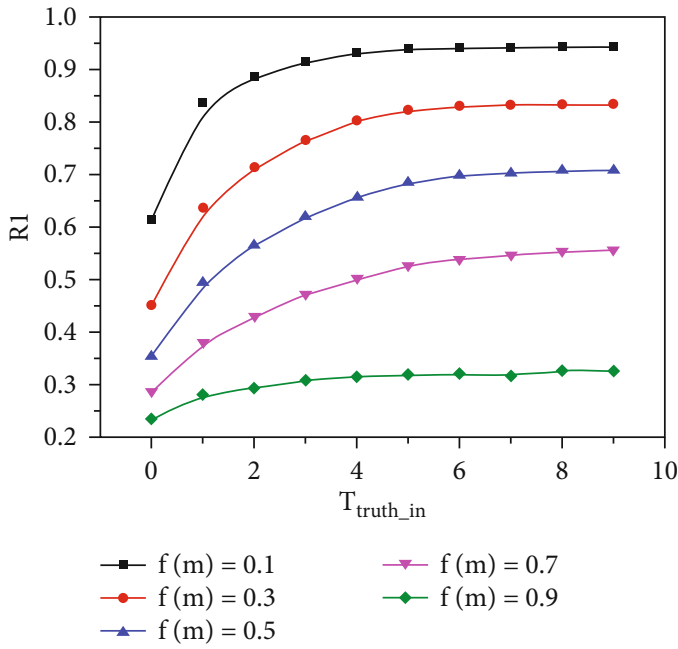

(a)

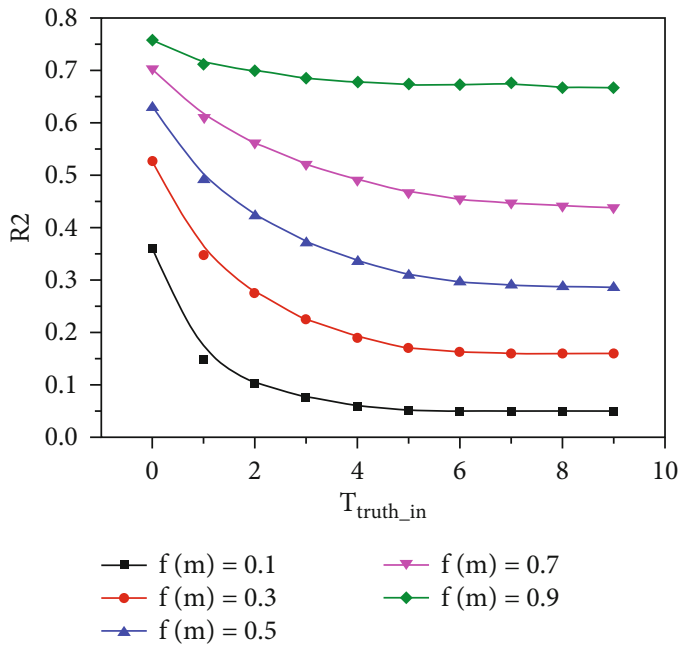

(b)

Figure 9: Densities of stifler1 and stifler2 for different values of $f(m)$ and $T_{\text {truth_in }}$ in the steady-state.

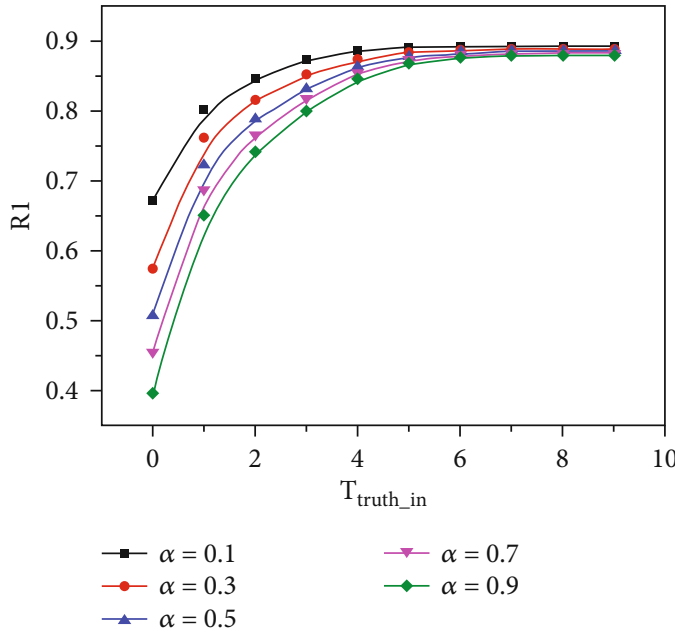

(a)

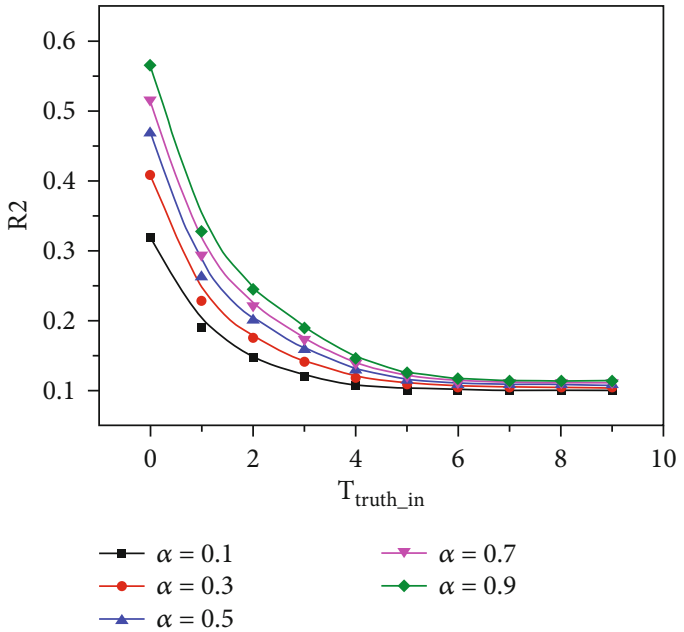

(b)

Figure 10: Densities of stifler1 and stifler2 for different values of $\alpha$ and $T_{\text {truth_in }}$ in the steady-state.

in the network is called $T_{\text {rumor_in }}$, and when $T_{\text {rumor_in }}=0$, the truth begins to spread. Figures 11 and 12 describe how the final size of stifler 1 and stifler2 change over $T_{\text {truth_in }}$ with different values of $f(m)$ and $\alpha$. From Figures 11 and 12, we can find that the earlier the rumor joins the network, the influence range of the rumor will be enlarged, and the truth will be reduced. In addition, comparing Figures 11 and 12, we can find that if the rumor can be spread at an earlier time (E.g., $T_{\text {rumor_in }}<2$ ), the influence range of the rumor will be reduced, and the truth will be enlarged significantly with increasing $f(m)$ or $\alpha$. But if the rumor begins to spread too late (E.g., $T_{\text {rumor_in }}>2$ ), the mechanisms of confrontation and discernibility will become ineffective, and the rumor cannot spread.

In summary, it can be concluded that the earlier release of the truth about the event by the government can significantly control the rumor. Even the truth of some well- known events has been already spread can suppress the appearance of the rumor. This also verifies the hypothesis of chapter Steady-state Analysis of Truth. Secondly, it is more effective to publish the truth in advance than after the rumor has appeared. This is because as shown in Figures 11 and 12, if the truth is spread earlier than the rumors, the rumors will be almost impossible to spread on the Internet. Thirdly, it is more important for the government to increase education and improve the ability of citizens to reveal the rumor than to increase the spread of the truth after the rumor occurs. Parameter $f(m)$ represents the ability of people to reveal the rumor, and from the comparison of Figures 9 and 10, improving the selfdiscrimination ability of citizens $(f(m))$ will permanently benefit the control of rumors and the spread of truth, while the confrontation mechanism may become ineffective (E.g., $\left.T_{\text {truth_in }}>5\right)$. 


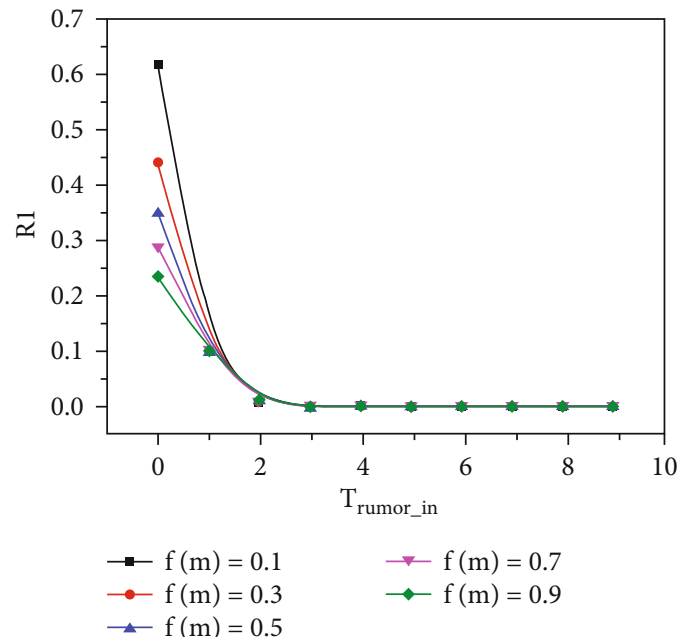

(a)

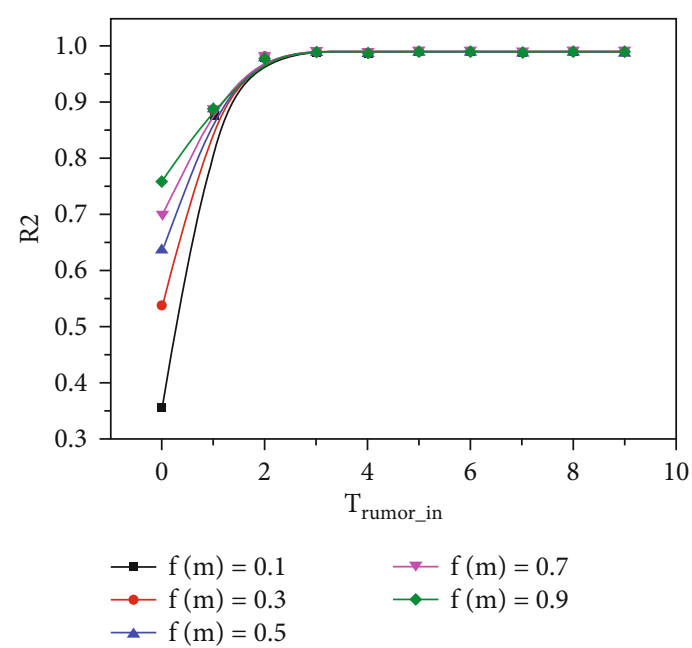

(b)

Figure 11: Densities of stifler1 and stifler2 for different values of $f(m)$ and $T_{\text {rumor_in }}$ in the steady-state.

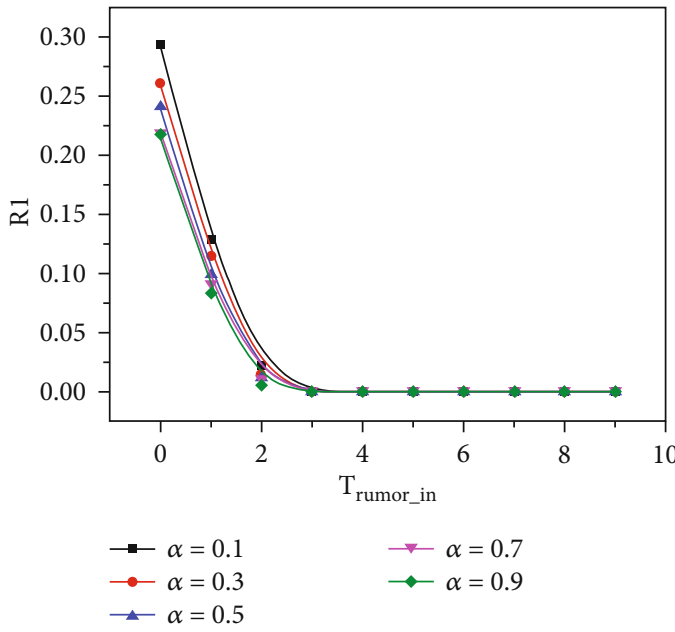

(a)

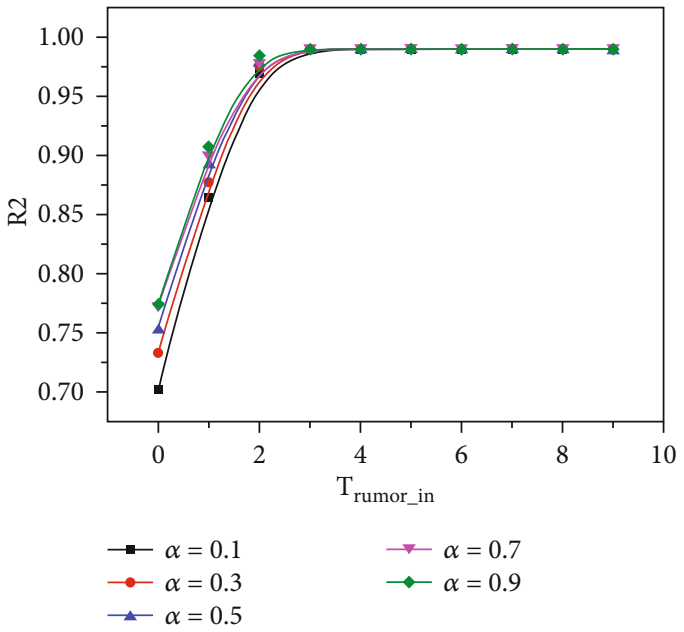

(b)

Figure 12: Densities of stifler 1 and stifler2 for different values of $\alpha$ and $T_{\text {rumor_in }}$ in the steady-state.

\section{Conclusions}

Rumors, as a special kind of information, usually damage reputation, cause social harm and can lead to a crisis for an individual or even an entire country. To minimize the damage caused by rumors, it is important to establish a reasonable model to study the process of rumor spreading. In this paper, we proposed an adjusted model in a generated heterogeneous network, called the 2SIH2R rumorspreading model, based on the traditional SIR model. The following significant results were obtained:

(1) Mechanisms of discernibility and confrontation were introduced to the model. Moreover, the 2SIH2R rumor-spreading model can be simplified into the traditional SIR and SEIR models under special circumstances
(2) After creating the mean-field equations to describe the dynamics of the 2SIH2R model, we obtained the spreading threshold under three cases by mathematical derivation. When only the rumor existed in the network, the spreading threshold was $\lambda_{1 c}=(<k$ $\left.>/<k^{2}>\right) \gamma_{1}-f(m) \eta /(1-f(m))$. When only the truth existed in the network, the spreading threshold was $\lambda_{2 c}=\left(\left\langle k>/<k^{2}\right\rangle\right) \gamma_{2}$. When there was one spreader 1 and one spreader 2 at $t=0$, the condition of the spreading threshold of the $2 \mathrm{SIH} 2 \mathrm{R}$ model was $\lambda_{1}(1-f(m))+\left(\theta_{1} / \theta_{1}+\theta_{2}\right) f(m) \eta>\left(<k>/<k^{2}>\right.$ ) $\gamma_{1}$ or $\lambda_{2}>\left(<k>/<k^{2}>\right) \gamma_{2}$. If the condition was not satisfied, the rumor or the truth could not spread widely in the heterogeneous network

(3) In a numerical simulation, we changed the strength of the discernible and confrontation mechanisms to 
study how they affected the impact of the rumor. The results show that under the same conditions, the greater are the mechanisms of discernibility and confrontation, the smaller is the influence of the rumor

(4) The earlier release of the truth about the event by the government can significantly control the rumor

(5) It is more effective to publish the truth in advance than after the rumor has appeared. In other words, through machine learning and public opinion monitoring technology in the era of big data, the government or authoritative media can spread some information or preissued warnings before a rumor appears. In this way, the influence of rumors can be reduced, and even the appearance of rumors can almost be stifled

(6) It is more important for the government to increase education and improve the ability of citizens to reveal the rumor than to increase the spread of the truth after the rumor occurs

\section{Data Availability}

The generated data used to support the findings of this study have not been made available because the data is randomly generated according to the rules.

\section{Conflicts of Interest}

The authors declare that there is no conflict of interest regarding the publication of this paper.

\section{Acknowledgments}

This paper is financially supported by the Beijing Municipal Natural Science Foundation (9202018), Fundamental Research Funds for the Central Universities (CUC21GZ005, CUC2000004, CUC19ZD002, 2018CUCTJ047), and Academic Research Project of China Federation of Radio and Television Association (2020ZGLH015).

\section{References}

[1] R. K. Garrett, "Troubling consequences of online political rumoring," Human Communication Research, vol. 37, no. 2, pp. 255-274, 2011.

[2] S. Galam, "Modelling rumors: the no plane pentagon french hoax case," Physica A, vol. 320, pp. 571-580, 2003.

[3] S. A. Thomas, "Lies, damn lies, and rumors: an analysis of collective efficacy, rumors, and fear in the wake of katrina," Sociological Spectrum, vol. 27, no. 6, pp. 679-703, 2007.

[4] N. DiFonzo, N. M. Robinson, J. M. Suls, and C. Rini, "Rumors about cancer: content, sources, coping, transmission, and belief," Journal of Health Communication, vol. 17, no. 9, pp. 1099-1115, 2012.

[5] H. J. Oh and H. Lee, "When do people verify and share health rumors on social media? The effects of message importance, health anxiety, and health literacy," Journal of Health Communication, vol. 24, no. 11, pp. 837-847, 2019.
[6] G. W. Allport and L. Postman, The Psychology of Rumor, American Psychological Association, 1947.

[7] Y. L. Zan, J. L. Wu, P. Li, and Q. Yu, "SICR rumor spreading model in complex networks: counterattack and self- resistance," Physica A, vol. 405, pp. 159-170, 2014.

[8] Q. M. Liu, T. Li, and M. Sun, "The analysis of an SEIR rumor propagation model on heterogeneous network," Physica A, vol. 469, pp. 372-380, 2017.

[9] D. J. Daley and D. G. Kendall, "Epidemics and Rumours," Nature, vol. 204, no. 4963, pp. 1118-1118, 1964.

[10] D. J. Daley and D. G. Kendall, "Stochastic rumours," IMA Journal of Applied Mathematics, vol. 1, no. 1, pp. 42-55, 1965.

[11] D. H. Zanette, "Critical behavior of propagation on smallworld networks," Physical Review E, vol. 64, no. 5, article 050901, 2001.

[12] D. H. Zanette, "Dynamics of rumor propagation on smallworld networks," Physical Review E, vol. 65, no. 4, article 041908, 2002.

[13] D. J. Watts and S. H. Strogatz, "Collective dynamics of 'smallworld' networks," Nature, vol. 393, no. 6684, pp. 440-442, 1998.

[14] Y. Moreno, M. Nekovee, and A. Pacheco, "Dynamics of rumor spreading in complex networks," Physical Review E, vol. 69, no. 6, article 066130, 2004.

[15] A. L. Barabási and R. Albert, "Emergence of scaling in random networks," Science, vol. 286, no. 5439, pp. 509-512, 1999.

[16] J. J. Wang, L. J. Zhao, and R. B. Huang, "SIRaRu rumor spreading model in complex networks," Physica A, vol. 398, pp. 4355, 2014.

[17] J. J. Wang, L. J. Zhao, and R. B. Huang, "2SI2R rumor spreading model in homogeneous networks," Physica A, vol. 413, pp. 153-161, 2014.

[18] L. Zhao, J. Wang, Y. Chen, Q. Wang, J. Cheng, and H. Cui, "SIHR rumor spreading model in social networks," Physica A, vol. 391, no. 7, pp. 2444-2453, 2012.

[19] L. Zhao, X. Qiu, X. Wang, and J. Wang, "Rumor spreading model considering forgetting and remembering mechanisms in inhomogeneous networks," Physica A, vol. 392, no. 4, pp. 987-994, 2013

[20] R. L. Rosnow, J. L. Esposito, and L. Gibney, "Factors influencing rumor spreading: replication and extension," Language of Communication, vol. 8, no. 1, pp. 29-42, 1988.

[21] L. A. Huo and Y. Y. Cheng, "Dynamical analysis of a IWSR rumor spreading model with considering the self- growth mechanism and indiscernible degree," Physica A, vol. 536, article 120940, 2019.

[22] L. L. Xia, G. P. Jiang, B. Song, and Y. R. Song, "Rumor spreading model considering hesitating mechanism in complex social networks," Physica A, vol. 437, pp. 295-303, 2015.

[23] A. Rizzo, M. Frasca, and M. Porfiri, "Effect of individual behavior on epidemic spreading in activity-driven networks," Physical Review E, vol. 90, no. 4, article 042801, 2014.

[24] K. Afassinou, "Analysis of the impact of education rate on the rumor spreading mechanism," Physica A, vol. 414, pp. 43-52, 2014.

[25] L. A. Huo, F. Ding, and Y. Y. Cheng, "Dynamic analysis of a SIbInIu, rumor spreading model in complex social network," Physica A, vol. 523, pp. 924-932, 2019.

[26] M. Askarizadeh, B. T. Ladani, and M. H. Manshaei, "An evolutionary game model for analysis of rumor propagation and 
control in social networks," Physica A, vol. 523, pp. 21-39, 2019.

[27] N. Zhang, H. Huang, B. Su, J. Zhao, and B. Zhang, "Dynamic 8-state ICSAR rumor propagation model considering official rumor refutation," Physica A, vol. 415, pp. 333-346, 2014.

[28] K. Ji, J. Liu, and G. Xiang, "Anti-rumor dynamics and emergence of the timing threshold on complex network," Physica A, vol. 411, pp. 87-94, 2014.

[29] M. Jiang, Q. Gao, and J. Zhuang, "Reciprocal spreading and debunking processes of online misinformation: a new rumor spreading-debunking model with a case study," Physica A, vol. 565, article 125572, 2021.

[30] Y. Wang, F. Qing, J.-P. Chai, and Y.-P. Ni, "Spreading dynamics of a 2 sih2r, rumor spreading model in the homogeneous network," Complexity, vol. 2021, Article ID 6693334, 9 pages, 2021.

[31] A. I. E. Hosni, K. Li, and S. Ahmad, "Analysis of the impact of online social networks addiction on the propagation of rumors," Physica A, vol. 542, article 123456, 2020.

[32] D. M. Fan, G. P. Jiang, Y. R. Song, and Y. W. Li, "Novel fake news spreading model with similarity on PSO-based networks," Physica A, vol. 549, article 124319, 2020.

[33] M. Yan, X. Lou, and Y. Wang, "Channel noise optimization of polar codes decoding based on a convolutional neural network," Wireless Communications and Mobile Computing, vol. 2021, Article ID 1434347, 10 pages, 2021.

[34] C. Y. Sang and S. G. Liao, "Modeling and simulation of information dissemination model considering user's awareness behavior in mobile social networks," Physica A, vol. 537, article 122639, 2020. 